This is the accepted manuscript made available via CHORUS. The article has been published as:

\title{
Strong Coupling between Magnons and Microwave Photons
} in On-Chip Ferromagnet-Superconductor Thin-Film Devices

Yi Li, Tomas Polakovic, Yong-Lei Wang, Jing Xu, Sergi Lendinez, Zhizhi Zhang, Junjia Ding, Trupti Khaire, Hilal Saglam, Ralu Divan, John Pearson, Wai-Kwong Kwok, Zhili Xiao, Valentine Novosad, Axel Hoffmann, and Wei Zhang Phys. Rev. Lett. 123, 107701 - Published 3 September 2019 DOI: 10.1103/PhysRevLett.123.107701 


\title{
Strong magnon-photon coupling in ferromagnet-superconducting resonator thin-film devices
}

\author{
Yi Li, ${ }^{1,2}$ Tomas Polakovic, ${ }^{3,4}$ Yong-Lei Wang, ${ }^{2,5}$ Jing Xu, ${ }^{2,6}$ Sergi Lendinez, ${ }^{2}$ Zhizhi Zhang, ${ }^{2,7}$ \\ Junjia Ding, ${ }^{2}$ Trupti Khaire, ${ }^{2}$ Hilal Saglam, ${ }^{2,8}$ Ralu Divan, ${ }^{9}$ John Pearson, ${ }^{2}$ Wai-Kwong \\ Kwok, ${ }^{2}$ Zhili Xiao, ${ }^{2,6}$ Valentine Novosad, ${ }^{2, *}$ Axel Hoffmann, ${ }^{2, \dagger}$ and Wei Zhang ${ }^{1,2, \ddagger}$ \\ ${ }^{1}$ Department of Physics, Oakland University, Rochester, MI 48309, USA \\ ${ }^{2}$ Materials Science Division, Argonne National Laboratory, Argonne, IL 60439, USA \\ ${ }^{3}$ Physics Division, Argonne National Laboratory, Argonne, IL 60439, USA \\ ${ }^{4}$ Department of Physics, Drexel University, Philadelphia, PA 19104, USA \\ ${ }^{5}$ Research Institute of Superconductor Electronics, \\ School of Electronic Science and Engineering, Nanjing University, 210093, Nanjing, China \\ ${ }^{6}$ Department of Physics, Northern Illinois University, Dekalb, IL 60115, USA \\ ${ }^{7}$ School of Optical and Electronic Information, Huazhong University of Science and Technology, Wuhan 430074, China \\ ${ }^{8}$ Department of Physics, Illinois Institute of Technology, Chicago IL 60616, USA \\ ${ }^{9}$ Center for Nanoscale Materials, Argonne National Laboratory, Argonne, IL 60439, USA
}

(Dated: May 13, 2019)

\begin{abstract}
We demonstrate strong magnon-photon coupling of a thin-film permalloy device fabricated on a coplanar superconducting resonator. A coupling strength of $0.152 \mathrm{GHz}$ and a cooperativity of 68 are found for a 30-nm-thick permalloy stripe. The coupling strength is tunable by rotating the biasing magnetic field or changing the volume of permalloy. We also observe an enhancement of magnonphoton coupling in the nonlinear regime of the superconducting resonator, which is mediated by the nucleation of dynamic flux vortices. Our results demonstrate a critical step towards future integrated hybrid systems for quantum magnonics and on-chip coherent information transfer.
\end{abstract}

Hybrid systems play a crucial role in quantum information processing [1-3]. In these systems, quantum states are coherently conveyed from one platform to another, with diverse carriers such as superconducting qubits, optical and microwave photons, individual atoms, ions, spin ensembles, and phonons [4-10]. These coherent transduction, which is represented by their mode hybridization [11], will be necessary to utilize the advantage of different state variables.

Recently, magnons have been considered as a new candidate for coherent information processing [12-26]. Magnons are the collective excitation of exchangecoupled spins in magnetic materials. They can conveniently couple to microwave photons via dipolar interaction. Especially, compared with paramagnetic spin ensembles which have been proposed as quantum memories [6, 7, 27-29], magnetic materials can provide much larger coupling strength and cooperativity, because they have spin densities 4 to 6 orders of magnitude higher than in spin ensembles [13]. This means magnons are capable of exchanging information with a much faster speed and for more cycles before losing coherency, while keeping small device dimensions. Coherent coupling between superconducting qubits and a single magnon has also been recently demonstrated $[18,24]$, showing the potential for magnons to conduct real quantum operations. Furthermore, with new advances in spin-charge interconversion $[30,31]$, the excitation of magnons in hybrid systems can be electrically detected via spin pumping $[19,21,32]$ and potentially other spin-transport phenomena $[33,34]$.

Despite the progress in magnon-photon hybrid system, which are predominantly centered on yttrium iron garnet (YIG) ferrimagnets [13-20, 22-26], the crucial step for extended development, i.e., on-chip integration and miniaturization are problematic for YIG because of the critical conditions in deposition and fabrication. In addition, maximizing coupling efficiency between magnons and microwave photons requires good proximity between magnetic spins and microwave resonator, which will be ideal if the magnetic devices are confined and directly fabricated on the resonator. Thus it is desired to explore alternative magnetic systems for large-scale magnon-based hybrid quantum systems.

In this work, we demonstrate an all-on-chip magnonphoton hybrid circuit with a permalloy $\left(\mathrm{Ni}_{80} \mathrm{Fe}_{20}, \mathrm{Py}\right)$ thin-film device directly fabricated on top of a coplanar superconducting resonator. On the magnon side, $\mathrm{Py}$ is a classical metallic ferromagnet with well-known magnetic properties and industry-friendly deposition requirement. It exhibits five times larger spin density than YIG and allows even larger coupling strengths. On the photon side, a coplanar superconducting resonator has a much smaller mode volume than a macroscopic microwave cavity along with a higher quality factor, which allows more concentrated and long-lived photons to couple with magnons. We achieve a strong magnon-photon coupling strength of $g / 2 \pi=0.152 \mathrm{GHz}$ and cooperativity of $C=68$ for a small volume of $\operatorname{Py}\left(V=400 \mu \mathrm{m}^{3}\right)$, along with a high coupling efficiency of $26.7 \mathrm{~Hz}$ per Bohr magneton. Furthermore, the coupling strength can be further enhanced by driving the superconducting resonator in the nonlinear regime, which is attributed to the creation of dynamic 
magnetic flux vortices. Our results suggest the combination of superconducting resonator and metallic ferromagnets can be a promising platform for investigating on-chip quantum magnonics and spintronics, and brings new potential for coherent manipulation and long-distance propagation of spin information.

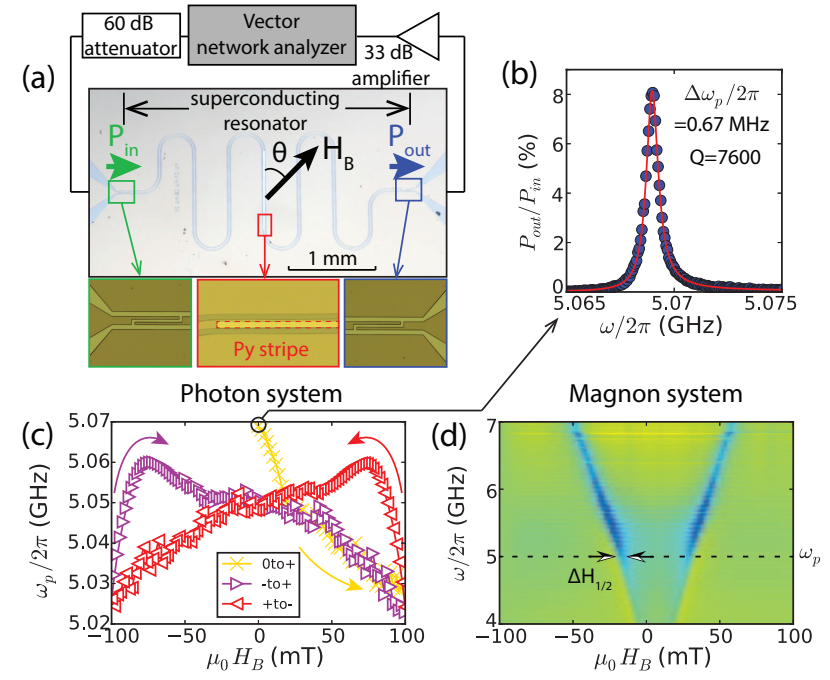

FIG. 1. (a) The microwave circuit of a NbN superconducting resonator with a Py stripe. The green (blue) and red boxes show the capacitive coupling to the external circuit and the permalloy stripe, respectively. (b) Microwave power transmission of an unloaded superconducting resonator measured at $P_{\text {in }}=-55 \mathrm{dBm}$ after zero-field cooling. (c) Hysteresis evolution of $\omega_{p}$ for (b). (d) Ferromagnetic resonance spectra of a Py stripe measured at $1.4 \mathrm{~K}$ [35], with the linewidth at $\omega_{p}$ marked by arrows.

Superconducting coplanar resonators were fabricated [36] from 200-nm-thick NbN films by photolithography and reactive ion etching, Fig. 1(a). The NbN films were deposited on undoped Si substrates via reactive sputtering technique at room temperature [37]. Subsequently, a 30-nm Py thin-film stripe with lateral dimensions of $14 \times 900 \mu \mathrm{m}^{2}$ was fabricated on top of the signal line of the resonator but electrically isolated from it by a 20-nm $\mathrm{MgO}$ insulating layer. The microwave response of the system is characterized by a vector network analyzer. Throughout the experiment the samples are cooled down to $1.4 \mathrm{~K}$, which is well below the superconducting transition temperature of the $\mathrm{NbN}$ resonator, $T_{c}=14 \mathrm{~K}$ [35].

The mode evolution of the individual magnon and photon systems are shown in Figs. 1(c) and (d), respectively, as a function of the in-plane magnetic field $\mu_{0} H_{B}$ along the stripline orientation $\left(\theta=0^{\circ}\right.$, defined in Fig. 1a). For the photon subsystem (without the Py stripe), the $\mathrm{NbN}$ resonator exhibits a sharp peak at $\omega_{p} / 2 \pi=5.069 \mathrm{GHz}$ with a full-width-half-maximum linewidth $\Delta \omega_{p} / 2 \pi=0.67 \mathrm{MHz}$, corresponding to a high quality-factor of $Q=7600$, see Fig. 1(b). The peak position corresponds to a dielectric constant, $\epsilon_{r} \sim 9.3$, similar to the value of 11.7 for Si. In addition, we obtain a hysteresis of $\omega_{p}$ by sweeping $\mu_{0} H_{B}$ (Fig. 1c). This behavior originates from the kinetic inductance variation from magnetic flux vortices in superconducting resonators [38]. For the magnon subsystem, we have fabricated an individual Py stripe on a coplanar waveguide and measured its broad-band ferromagnetic resonance at $1.4 \mathrm{~K}$ [35]. Two branches of resonance absorption are symmetrically located on the positive and negative fields, as shown in Fig. 1(d). The small field offset, $\sim 5 \mathrm{mT}$, comes from the hysteresis of the superconducting magnet coils. From Fig. 1(d), we can determine the linewidth of the ferromagnetic resonance as $\mu_{0} \Delta H_{1 / 2}=6.0 \mathrm{mT}$ at the frequency of $\omega_{p}$. This corresponds to a magnon damping rate of $\kappa_{m} / 2 \pi=(\gamma / 2 \pi) \mu_{0} \Delta H_{1 / 2}=0.178 \mathrm{GHz}$, in which $\gamma / 2 \pi=\left(g_{\text {eff }} / 2\right) \cdot 28 \mathrm{GHz} / \mathrm{T}$ is the gyromagnetic ratio and $g_{\text {eff }}=2.12$ is the g-factor for $\mathrm{Py}$.

We then turn to the magnon-photon hybridization. Before the Py deposition, the superconducting resonator exhibits a continuous spectra when $\mu_{0} H_{B}$ is swept from $-100 \mathrm{mT}$ to $100 \mathrm{mT}$, as shown in Fig. 2(a). After Py deposition, two avoided crossings appear symmetrically at positive and negative $\mu_{0} H_{B}$ (Fig. 2b). The mode anti-crossing indicates a strong coupling between the resonator photons and the ferromagnetic magnons. We also observe a broad resonance at $5.00 \mathrm{GHz}$ for Fig. 2(a) and $4.88 \mathrm{GHz}$ for Fig. 2(b), both independent of $\mu_{0} H_{B}$. They come from spurious resonances of the microwave circuits and are not relevant to the mode hybridization. The extracted peak positions $\left(\omega_{m p}\right)$ and linewidths $\left(\Delta \omega_{m p}\right)$ of the spectra are summarized in Figs. 2(c) and (d), respectively. A frequency offset of $0.12 \mathrm{GHz}$ between Figs. 2(a) and (b) has been taken into account due to the local impedance change from the additional Py stripe. As shown in Fig. 2(c), the two anti-crossings are located where the two magnon branches of the Py stripe intersect with the photon mode of the resonator, which clearly indicates the strong magnon-photon coupling.

The transmitted power of the hybrid system can be expressed as $[13-15,19]$ :

$$
\frac{P_{\text {out }}}{P_{\text {in }}}=\frac{\kappa_{R}}{i\left(\omega_{p}-\omega\right)+\kappa_{p}+\frac{g^{2}}{i\left(\omega_{m}-\omega\right)+\kappa_{m}}}
$$

where $\kappa_{R}$ is the capacitive coupling of the resonator to the external circuits and $g$ is the magnon-photon coupling strength. $\kappa_{p}$ is increased from Fig. 1 (b) to $\kappa_{p} / 2 \pi=2.0$ $\mathrm{MHz}$ with the additional Py load, see Fig. 2(d) at high fields. Fig. 2(c) overlays the fits to the eigenmode solution of Eq. (1) on top of the extracted $\omega_{p}$ from Fig. 2(b), with a single fit parameter $g / 2 \pi=0.152 \mathrm{GHz}$ [35]. Note the large value of $g$ despite the small ferromagnetic volume with merely $30-\mathrm{nm}$ of Py. To understand the origin, the coupling strength is expressed as $g=g_{0} \sqrt{N}$ where $N$ is the total number of spins and $g_{0}=\gamma \sqrt{\mu_{0} \hbar \omega_{p} / V_{c}}$ is the 

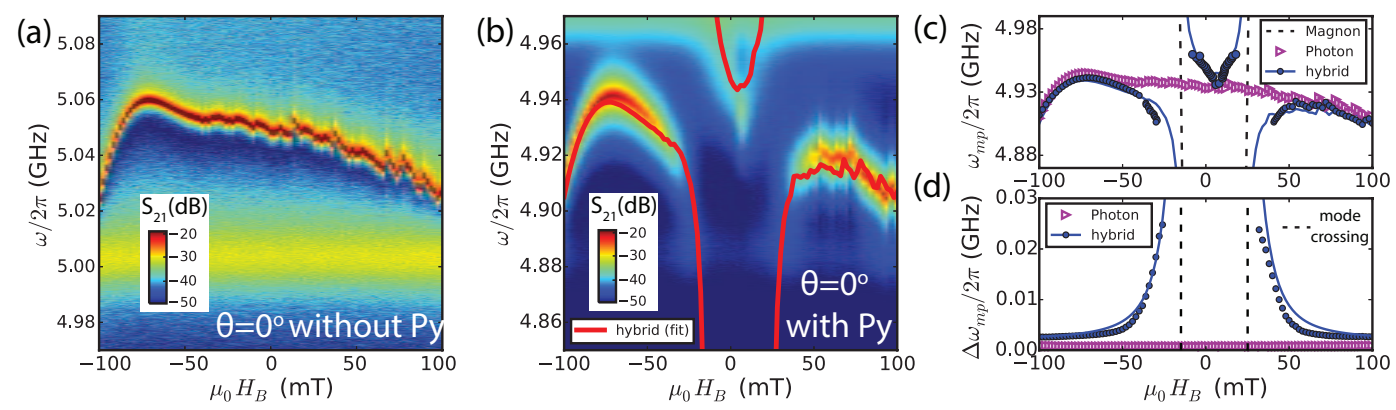

FIG. 2. Characterization of a $30-\mathrm{nm}$ Py stripe $(\mathrm{L}=900 \mu \mathrm{m})$ coupled to a $\mathrm{NbN}$ superconducting resonator, measured at $P_{\text {in }}=-55 \mathrm{dBm}$ and $\theta=0^{\circ}$. (a-b) Microwave transmission spectra $S_{21}=10 \log \left(P_{\text {out }} / P_{\text {in }}\right)$ of (a) the unloaded resonator and (b) the resonator loaded with the Py stripe. (c) Extracted $\omega_{m p}$ and (d) $\Delta \omega_{m p}$ from (a-b). In (c) the photon modes have been shifted by $-0.12 \mathrm{GHz}$ to match the hybrid modes. Dashed lines denote the magnon modes. Solid blue and red curves denote the fits.

coupling strength of the superconducting resonator to a single Bohr magneton. Here $\hbar$ is the Planck constant, and $V_{c}$ is the mode volume of the resonator. Using the dimensions of the Py stripe and $\mu_{0} M_{s}=1 \mathrm{~T}$ for the Py saturation magnetization, we calculate $N=3.25 \times 10^{13}$ and $g_{0}=26.7 \mathrm{~Hz}$ from the experiment. We highlight that our $g_{0}$ is three orders of magnitude larger compared with using a macroscopic cavity $[14,15]$. It comes from the small mode volume $V_{c} \sim 0.0051 \mathrm{~mm}^{3}$ for the coplanar resonator and indicates the significance of having a localized and concentrated photon mode volume to reach a strong coupling strength. It is worthwhile to note a few different planar resonator designs such as split-ring $[17,39,40]$ and lumped-element resonators [41, 42]. The former allows an optimal filling of thin-film magnetic materials in the resonator, with $g / 2 \pi$ close to $1 \mathrm{GHz}$; the latter has the highest $g_{0}$ by further reducing the mode volume. In addition, compared with the similar superconducting resonator structure coupled to a YIG slab [13] $\left(g / 2 \pi=0.45 \mathrm{GHz}, g_{0} / 2 \pi=2.5 \mathrm{~Hz}\right.$ and $\left.N=4 \times 10^{16}\right)$, our $g_{0}$ is one order of magnitude larger because the Py device is in good proximity to the resonator and maintains optimal coupling efficiency. This yields a comparable $g$ of Py stripe but with three orders of magnitude less number of total spins than in the YIG slab. A large cooperativity of the hybrid system as $C=g^{2} / \kappa_{m} \kappa_{p}=68$ is obtained, which is a promising feature of coherent information exchange between photons and magnons in $\mathrm{Py}$.

In addition to the frequency shift, we also observe a linewidth variation for the hybrid modes [19]. In Fig. $2(\mathrm{~d})$, when $\mu_{0} H_{B}$ is close to the anti-crossing regime, $\Delta \omega_{m p}$ quickly increases from the photon damping rate $\kappa_{p} / 2 \pi=2 \mathrm{MHz}$ and approaches the magnon damping rate $\kappa_{m}$. This is due to the mixing of relaxation channels when the magnon and photon modes are hybridized, see the Supplemental Materials [35]. We plot the theoretical prediction in Fig. 2(d) with the same input values of $g, \kappa_{p}$ and $\kappa_{m}$ in Fig. 2(c), and the linewidth of the hybrid modes can be reproduced. Between the two mode-crossing gaps (between $-25 \mathrm{mT}$ and $+32 \mathrm{mT}$ ) the hybrid modes are influenced by the saturation state of the Py stripe, which significantly deviate from the macrospin model and are not shown.

The coupling strength $g$ is tunable by changing the dipolar coupling efficiency between magnons and photons as well as changing the total number of spins in Py. Figs. 3(a-d) show the microwave transmission spectra of the same device in Fig. 2 at different $\theta$. As $\theta$ deviates from $0^{\circ}$, the mode anti-crossing becomes smaller and disappears at $90^{\circ}$. This is due to the change of dipolar coupling energy, $E=\mu_{0} M_{\perp} h_{r f} \cos \theta$, where the transverse components of the dynamic magnetization $M_{\perp}$ and microwave field $h_{r f}$ are no longer parallel and become orthogonal when $\theta=90^{\circ}$. The extracted $g$ can be modeled by a cosine function of $\theta$ (red curve) in Fig. 3(e). In addition, there are two additional observations in Figs. $3(\mathrm{a}-\mathrm{d})$ : the mode anti-crossing moves towards the lower biasing fields, and a spectral gap appears near $\mu_{0} H_{B}=0$ $\mathrm{mT}$ for $\theta=90^{\circ}$. They are due to the shape anisotropy of the Py stripe [43], which pins the Py magnetization along $\theta=0^{\circ}$ at low fields. To vary the total number of spins for magnons, we have fabricated a new series of Py stripes with different lengths $(L)$ and thicknesses $(t)$ and show their transmission spectra in Fig. 3(g-i). As the coupling strength is reduced, the mode hybridization becomes weaker and for the smallest Py volume in Fig. 3(i), the spectra go into the Purcell regime [15] as $g$ becomes significantly smaller than $\kappa_{m}$. In Fig. $3(\mathrm{j})$ a linear fit to $g=g_{0} \sqrt{N}$ is shown with a red line with an extracted $g_{0} / 2 \pi=21.4 \mathrm{~Hz}$, which is close to the value obtained in Fig. 2.

The evolution of $\Delta \omega_{m p}$ also changes accordingly for different magnon-photon coupling conditions. In Fig. $3(\mathrm{f})$ when the biasing field is far away from the anticrossing regime $\left(\mu_{0} H_{B}= \pm 100 \mathrm{mT}\right), \Delta \omega_{m p}$ shows a consistent value of $2.5 \mathrm{MHz}$ for the same Py device at different $\theta$. As $H_{B}$ approaches the anti-crossing conditions, $\Delta \omega_{m p}$ increases much slower for larger $\theta$, because 


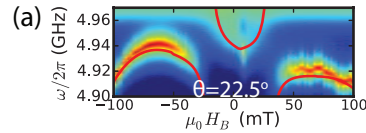

(b)

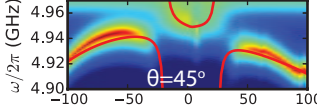

(c)

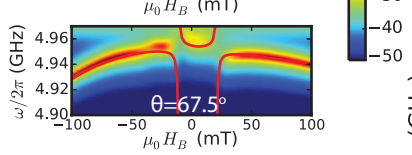

(d)
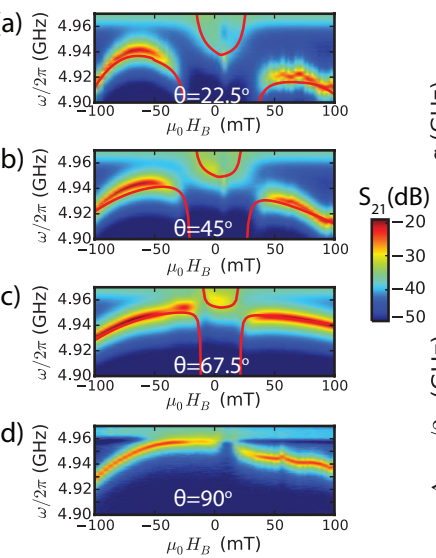
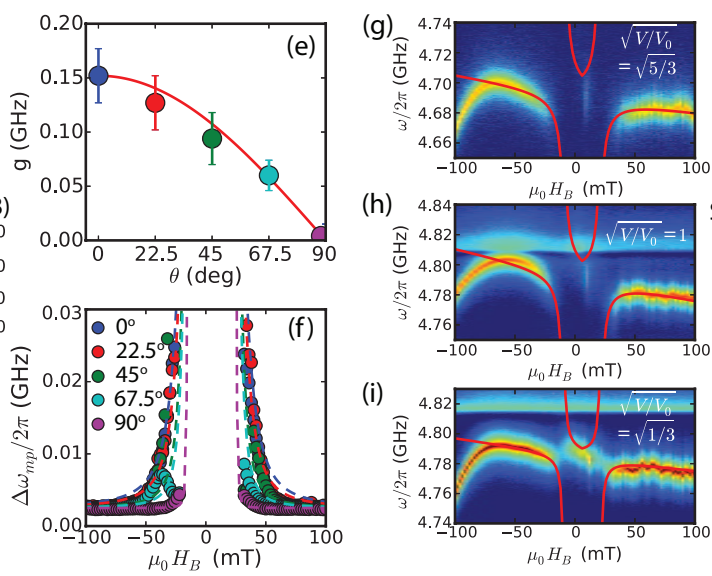

(h)
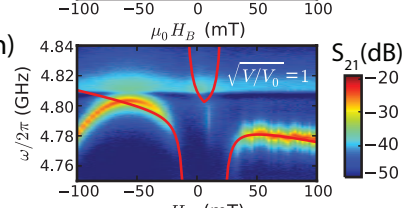

(i)
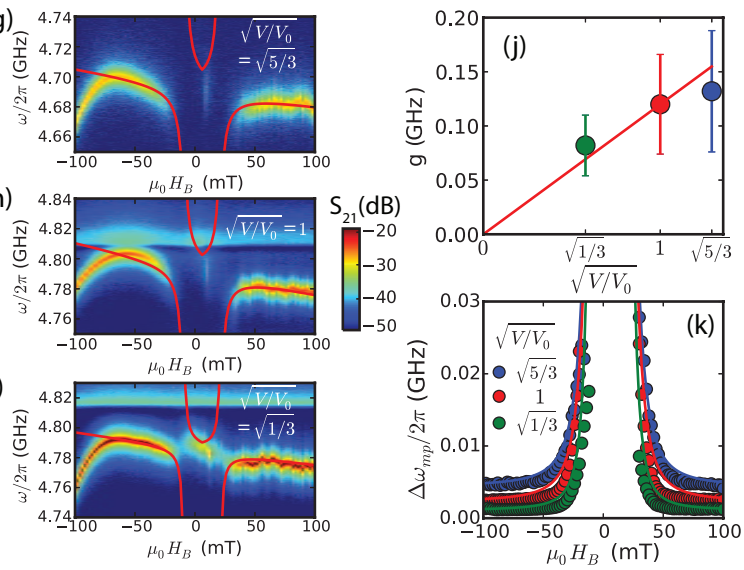

FIG. 3. Tunable magnon-photon coupling. (a-d) Microwave transmission spectra of the NbN superconducting resonator loaded with a $\operatorname{Py}(30 \mathrm{~nm})$ stripe with $L=900 \mu \mathrm{m}$, from $\theta=22.5^{\circ}$ to $90^{\circ}$. (e) Extracted coupling strength $g$ as a function of $\theta$ with the fit. (f) $\Delta \omega_{p}$ as a function of $\mu_{0} H_{B}$ with the dashed fits. (g-i) Transmission spectra for different Py stripes: (g) $t=50 \mathrm{~nm}$, $L=900 \mu \mathrm{m}$; (h) $t=30 \mathrm{~nm}, L=900 \mu \mathrm{m}$; (i) $t=30 \mathrm{~nm}, L=300 \mu \mathrm{m}$. (j) Extracted $g$ as a function of $\sqrt{V / V_{0}}$, where $V_{0}$ denotes the volume of $\operatorname{Py}(30 \mathrm{~nm})$ stripe with $L=900 \mu \mathrm{m}$. (k) $\Delta \omega_{p}$ as a function of $\mu_{0} H_{B}$, with the fitting curve also plotted.

the coupling strength is decreasing. This trend can be theoretically reproduced in dashed curves by taking different values of $g$ from Fig. 3(e) in the theoretical model [35]. In Fig. 3(k), due to the variation of the dielectric loss from $\mathrm{Py}$, the values of $\kappa_{p}$ are different, as $4.4 \mathrm{MHz}$, $2.3 \mathrm{MHz}$ and $1.1 \mathrm{MHz}$ for Figs. 3(g) to (i), respectively. By accounting for this $\kappa_{p}$ variation, the linewidths can be also well fitted for different Py volumes.

Next, we show that the photon mode in the hybrid system can easily go into the nonlinear regime. This concept has been used for high-fidelity quantum operations [4446]. In Fig. 4(a), we show the output lineshapes of the superconducting resonator loaded with a $\mathrm{Py}(50 \mathrm{~nm})$ stripe from $P_{i n}=-15 \mathrm{dBm}$ to $5 \mathrm{dBm}$, at $\mu_{0} H_{B}=100 \mathrm{mT}$ and $\theta=0^{\circ}$. A nonlinear shift of the peak position towards the lower frequency is observed, with a critical power of
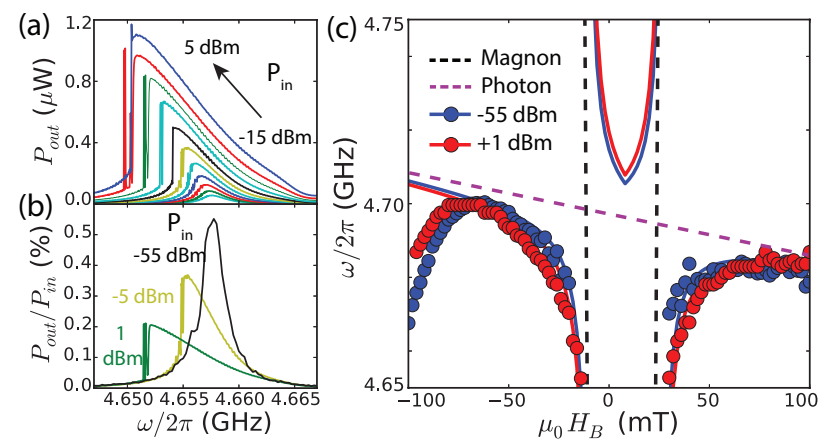

FIG. 4. Magnon-photon coupling in the nonlinear regime. (a) Nonlinear resonance lineshapes from $P_{\text {in }}=-15 \mathrm{dBm}$ to $P_{i n}=5 \mathrm{dBm}$ with a step of $2 \mathrm{~dB}$ for $\mathrm{Py}(50 \mathrm{~nm})$ stripe with $L=900 \mu \mathrm{m}$. (b) $P_{\text {out }} / P_{\text {in }}$ for $P_{\text {in }}=-55 \mathrm{dBm},-5 \mathrm{dBm}$ and $1 \mathrm{dBm}$. (c) Comparison of peak positions of the hybrid modes between $P_{i n}=1 \mathrm{dBm}$ and $-55 \mathrm{dBm}$.
$P_{c}=-5 \mathrm{dBm}$ for the lineshape to reach a vertical slope (Fig. 4b). This critical power is well below the typical threshold power for the magnon system alone to reach the nonlinear regime [47]. The origin of the nonlinearity is the kinetic inductance variation of flux vortices [48] in the $\mathrm{NbN}$ resonator, which leads to frequency downshifts as also observed in Fig. 1(c) at increasing $H_{B}$. We note that such vortex-induced nonlinearity in the superconducting resonator can be extended to the variation of a single vortex flux [49], which shows potentials for conducting operation in the quantum limit. The dynamics of magnetic flux vortices also leads to an enhanced photon damping rate $\kappa_{p}$ [50], reflected by the reduction of maximal value of $P_{\text {out }} / P_{\text {in }}$ in Fig. 4(b).

Accompanied by the resonator nonlinearity, we also observe an enhanced magnon-photon coupling. In Fig. 4(c) we show the extracted peak positions of the hybrid mode for the 50-nm Py stripe. In the vicinity of the anticrossing regimes ( $\mu_{0} H_{B}$ close to the magnon branch), the peaks with $P_{i n}=1 \mathrm{dBm}$ show a stronger mode repelling compared with $P_{\text {in }}=-55 \mathrm{dBm}$. Fitting the data to Eq. (1) yields a coupling strength of $0.158 \mathrm{GHz}$ at $1 \mathrm{dBm}$ input, which is $14 \%$ larger than the value of $0.139 \mathrm{GHz}$ at $-55 \mathrm{dBm}$, in Fig. 3(j). This coupling enhancement is likely caused by the Meissner field trapping from the dynamic flux vortices [51], which will influence the distribution of the magnetic field at the superconducting stripline and thus change the dipolar coupling strength with the Py magnon system. Therefore, the magnonphoton coupling may be also used as an effective means for detecting flux vortex dynamics in hybrid superconducting devices.

In conclusion, we have demonstrated a new hybrid platform consisting of a superconducting resonator and a ferromagnetic device integrated on a Si substrate. We ob- 
tained a large magnon-photon coupling strength of 0.152 $\mathrm{GHz}$ and a cooperativity of 68 for a 30-nm-thick Py stripe. We also show that the superconducting resonator can easily reach the nonlinear regime, in which the efficiency of the magnon-photon coupling is improved. Our results indicate that magnon-photon hybrid systems are promising as a high-speed and coherent transducer for realizing circuit quantum electrodynamics [24], in microscopic magnetic devices that are compatible with on-chip designs. In spintronics, magnon-photon hybrid systems allow for exploring novel physics [52-54] and provide a means to transmit spin excitations coherently at long distance with photon excitations [55, 56], outperforming the currently-reported micro-meter propagation using pure spin currents [57] or spin waves [58]. Thus this demonstration of strong magnon-photon coupling in planar thin-film devices provides a crucial stepping stone for the development of more complex quantum information systems.

We acknowledge helpful discussions with Andy Kent and Xufeng Zhang. Wei Zhang acknowledges support from the U.S. National Science Foundation under Grant No. DMR-1808892 and DOE-Visiting Faculty Program. Work at Argonne, including thin films synthesis, device fabrications, and low-temperature measurements, were supported by the U.S. Department of Energy (DOE), Office of Science, Materials Science and Engineering Division. The use of Center for Nanoscale Materials for lithographic processing is supported by DOE-BES, under Contract No. DE-AC02-06CH11357.

* novosad@anl.gov

† hoffmann@anl.gov

¥ weizhang@oakland.edu

[1] M. Wallquist, K. Hammerer, P. Rabl, M. Lukin, and P. Zoller, Phys. Scr. 2009, 014001 (2009).

[2] Z.-L. Xiang, S. Ashhab, J. Q. You, and F. Nori, Rev. Mod. Phys. 85, 623 (2013).

[3] G. Kurizki, P. Bertet, Y. Kubo, K. Mølmer, D. Petrosyan, P. Rabl, and J. Schmiedmayer, Proc. Natl. Acad. Sci. 112, 3866 (2015).

[4] A. Wallraff, D. I. Schuster, A. Blais, L. Frunzio, R.-S. Huang, J. Majer, S. M. Kumar, S. abd Girvin, and R. J. Schoelkopf, Nature 431, 162 (2004).

[5] J. Verdú, H. Zoubi, C. Koller, J. Majer, H. Ritsch, and J. Schmiedmayer, Phys. Rev. Lett. 103, 043603 (2009).

[6] D. I. Schuster, A. P. Sears, E. Ginossar, L. DiCarlo, L. Frunzio, J. J. L. Morton, H. Wu, G. A. D. Briggs, B. B. Buckley, D. D. Awschalom, and R. J. Schoelkopf, Phys. Rev. Lett. 105, 140501 (2010).

[7] Y. Kubo, F. R. Ong, P. Bertet, D. Vion, V. Jacques, D. Zheng, A. Dréau, J.-F. Roch, A. Auffeves, F. Jelezko, J. Wrachtrup, M. F. Barthe, P. Bergonzo, and D. Esteve, Phys. Rev. Lett. 105, 140502 (2010).

[8] X. Zhu, S. Saito, A. Kemp, K. Kakuyanagi, S.-i. Karimoto, H. Nakano, W. J. Munro, Y. Tokura, M. S. Everitt,
K. Nemoto, M. Kasu, N. Mizuochi, and K. Semba, Nature 478, 221 (2011).

[9] E. Verhagen, S. Deléglise1, S. Weis, A. Schliesser, and T. J. Kippenberg, Nature 482, 63 (2012).

[10] J. J. Viennot, M. C. Dartiailh, A. Cottet, and T. Kontos, Science 349, 408 (2015).

[11] A. Blais, R.-S. Huang, A. Wallraff, S. M. Girvin, and R. J. Schoelkopf, Phys. Rev. A 69, 062320 (2004).

[12] O. O. Soykal and M. E. Flatté, Phys. Rev. Lett. 104, 077202 (2010).

[13] H. Huebl, C. W. Zollitsch, J. Lotze, F. Hocke, M. Greifenstein, A. Marx, R. Gross, and S. T. B. Goennenwein, Phys. Rev. Lett. 111, 127003 (2013).

[14] Y. Tabuchi, S. Ishino, T. Ishikawa, R. Yamazaki, K. Usami, and Y. Nakamura, Phys. Rev. Lett. 113, 083603 (2014).

[15] X. Zhang, C.-L. Zou, L. Jiang, and H. X. Tang, Phys. Rev. Lett. 113, 156401 (2014).

[16] M. Goryachev, W. G. Farr, D. L. Creedon, Y. Fan, M. Kostylev, and M. E. Tobar, Phys. Rev. Applied 2, 054002 (2014).

[17] B. Bhoi, T. Cliff, I. S. Maksymov, M. Kostylev, R. Aiyar, N. Venkataramani, S. Prasad, and R. L. Stamps, J. Appl. Phys. 116, 243906 (2014).

[18] Y. Tabuchi, S. Ishino, A. Noguchi, T. Ishikawa, R. Yamazaki, K. Usami, and Y. Nakamura, Science 349, 405 (2015).

[19] L. Bai, M. Harder, Y. P. Chen, X. Fan, J. Q. Xiao, and C.-M. Hu, Phys. Rev. Lett. 114, 227201 (2015).

[20] D. Zhang, X. M. Wang, T. F. Li, X. Q. Luo, W. Wu, F. Nori, and J. Q. You, npj Quantum Inf. 1, 15014 (2015).

[21] Y. Cao, P. Yan, H. Huebl, S. T. B. Goennenwein, and G. E. W. Bauer, Phys. Rev. B 91, 094423 (2015).

[22] N. J. Lambert, J. A. Haigh, and A. J. Ferguson, J. Appl. Phys. 117, 053910 (2015).

[23] N. Kostylev, M. Goryachev, and M. E. Tobar, Appl. Phys. Lett. 108, 062402 (2016).

[24] D. Lachance-Quirion, Y. Tabuchi, S. Ishino, A. Noguchi, T. Ishikawa, R. Yamazaki, and Y. Nakamura, Science Advances 3 (2017), 10.1126/sciadv.1603150.

[25] R. G. E. Morris, A. F. van Loo, S. Kosen, and A. D. Karenowska, Sci. Rep. 7, 11511 (2017).

[26] I. Boventer, M. Pfirrmann, J. Krause, Y. Schön, M. Kläui, and M. Weides, Phys. Rev. B 97, 184420 (2018).

[27] A. Imamoğlu, Phys. Rev. Lett. 102, 083602 (2009).

[28] J. H. Wesenberg, A. Ardavan, G. A. D. Briggs, J. J. L. Morton, R. J. Schoelkopf, D. I. Schuster, and K. Mølmer, Phys. Rev. Lett. 103, 070502 (2009).

[29] S. Probst, H. Rotzinger, S. Wünsch, P. Jung, M. Jerger, M. Siegel, A. V. Ustinov, and P. A. Bushev, Phys. Rev. Lett. 110, 157001 (2013).

[30] A. Hoffmann, IEEE Trans. Magns. 49, 5172 (2013).

[31] J. Sinova, S. O. Valenzuela, J. Wunderlich, C. H. Back, and T. Jungwirth, Rev. Mod. Phys. 87, 1213 (2015).

[32] O. Mosendz, J. E. Pearson, F. Y. Fradin, G. E. W. Bauer, S. D. Bader, and A. Hoffmann, Phys. Rev. Lett. 104, 046601 (2010).

[33] L. Liu, T. Moriyama, D. C. Ralph, and R. A. Buhrman, Phys. Rev. Lett. 106, 036601 (2011).

[34] H. Nakayama, M. Althammer, Y.-T. Chen, K. Uchida, Y. Kajiwara, D. Kikuchi, T. Ohtani, S. Geprägs, M. Opel, S. Takahashi, R. Gross, G. E. W. Bauer, S. T. B. 
Goennenwein, and E. Saitoh, Phys. Rev. Lett. 110, 206601 (2013).

[35] See the Supplemental Information for details.

[36] M. Göppl, A. Fragner, M. Baur, R. Bianchetti, S. Filipp, J. M. Fink, P. J. Leek, G. Puebla, L. Steffen, and A. Wallraff, J. Appl. Phys. 104, 113904 (2008).

[37] T. Polakovic, S. Lendinez, J. E. Pearson, A. Hoffmann, V. Yefremenko, C. L. Chang, W. Armstrong, K. Hafidi, G. Karapetrov, and V. Novosad, APL Materials 6, 076107 (2018).

[38] D. Bothner, T. Gaber, M. Kemmler, D. Koelle, R. Kleiner, S. Wünsch, and M. Siegel, Phys. Rev. B 86, 014517 (2012).

[39] B. Bhoi, B. Kim, J. Kim, Y.-J. Cho, and S.-K. Kim, Sci. Rep. 7, 11930 (2017).

[40] L. V. Abdurakhimov, S. Khan, N. A. Panjwani, J. D. Breeze, M. Mochizuki, S. Seki, Y. Tokura, J. J. L. Morton, and H. Kurebayashi, Phys. Rev. B 99, 140401 (2019).

[41] A. Bienfait, J. J. Pla, Y. Kubo, M. Stern, X. Zhou, C. C. Lo, C. D. Weis, T. Schenkel, . M. L. W. Thewalt, D. Vion, D. Esteve, B. Julsgaard, K. Mølmer, J. J. L. Morton, and P. Bertet, Nature Nanotech. 11, 253 (2016).

[42] C. Eichler, A. J. Sigillito, S. A. Lyon, and J. R. Petta, Phys. Rev. Lett. 118, 037701 (2017).

[43] Y. Li, Y. Lu, and W. E. Bailey, Journal of Applied Physics 113, 17B506 (2013).

[44] B. Yurke and E. Buks, J. Lightwave Technol. 24, 5054 (2006).

[45] F. Mallet, F. R. Ong, A. Palacios-Laloy, F. Nguyen, P. Bertet, D. Vion, and D. Esteve, Nature Phys. 5, 791 (2009).

[46] F. R. Ong, M. Boissonneault, F. Mallet, A. Palacios-
Laloy, A. Dewes, A. C. Doherty, A. Blais, P. Bertet, D. Vion, and D. Esteve, Phys. Rev. Lett. 106, 167002 (2011).

[47] Y.-P. Wang, G.-Q. Zhang, D. Zhang, T.-F. Li, C.-M. Hu, and J. Q. You, Phys. Rev. Lett. 120, 057202 (2018).

[48] M. A. Golosovsky, H. J. Snortland, and M. R. Beasley, Phys. Rev. B 51, 6462 (1995).

[49] I. Nsanzineza and B. L. T. Plourde, Phys. Rev. Lett. 113, 117002 (2014).

[50] C. Song, T. W. Heitmann, M. P. DeFeo, K. Yu, R. McDermott, M. Neeley, J. M. Martinis, and B. L. T. Plourde, Phys. Rev. B 79, 174512 (2009).

[51] M. Taupin, I. Khaymovich, M. Meschke, A. Melnikov, and J. P. Pekola, Nature Communi. 7, 10977 (2016).

[52] M. Harder, Y. Yang, B. M. Yao, C. H. Yu, J. W. Rao, Y. S. Gui, R. L. Stamps, and C.-M. Hu, Phys. Rev. Lett. 121, 137203 (2018).

[53] B. Bhoi, B. Kim, S.-H. Jang, J. Kim, J. Yang, Y.-J. Cho, and S.-K. Kim, Phys. Rev. B 99, 134426 (2019).

[54] I. Boventer, C. Dörflinger, T. Wolz, R. Macêdo, R. Lebrun, M. Kläui, and M. Weides, arXiv 1904.00393.

[55] X. Zhang, C.-L. Zou, N. Zhu, F. Marquardt, L. Jiang, and H. X. Tang, Nature Communi. 6, 8914 (2015).

[56] L. Bai, M. Harder, P. Hyde, Z. Zhang, C.-M. Hu, Y. P. Chen, and J. Q. Xiao, Phys. Rev. Lett. 118, 217201 (2017).

[57] S. O. Valenzuela and M. Tinkham, Nature 442, 176 (2006).

[58] Y. Kajiwara, K. Harii, S. Takahashi, J. Ohe, K. Uchida, M. Mizuguchi, H. Umezawa, H. Kawai, K. Ando, K. Takanashi, M. S., and E. Saitoh, Nature Physics 464, 262 (2010). 